\title{
Density functional theory study of the magnetic moment of solute Mn in bec Fe
}

\author{
D. J. M. King, ${ }^{1,}{ }^{*}$ S. C. Middleburgh,${ }^{2}$ P. A. Burr, ${ }^{3}$ T. M. Whiting, ${ }^{1}$ P. C. Fossati,${ }^{1}$ and M. R. Wenman ${ }^{1}$ \\ ${ }^{1}$ Centre for Nuclear Engineering, Imperial College London, South Kensington, London SW7 2AZ, United Kingdom \\ ${ }^{2}$ Nuclear Futures Institute, Bangor University, Dean Street, Bangor, Wales LL57 1UT, United Kingdom \\ and Westinghouse Electric Sweden AB, SE-721 63 Västerås, Sweden \\ ${ }^{3}$ School of Electrical Engineering, University of New South Wales, Kensington, 2052 NSW, Australia
}

(Received 18 April 2018; published 20 July 2018)

\begin{abstract}
An unexplained discrepancy exists between the experimentally measured and theoretically calculated magnetic moments of $\mathrm{Mn}$ in $\alpha$-Fe. In this study, we use density functional theory to suggest that this discrepancy is likely due to the local strain environment of a Mn atom in the Fe structure. The ferromagnetic coupling, found by experiment, was shown to be metastable and could be stabilized by a $2 \%$ hydrostatic compressive strain. The effects of Mn concentration, vacancies, and interstitial defects on the magnetic moment of Mn are also discussed. It was found that the ground-state, antiferromagnetic (AFM) coupling of Mn to Fe requires long-range tensile relaxations of the neighboring atoms along $\langle 111\rangle$ which is hindered in the presence of other Mn atoms. Vacancies and Fe interstitial defects stabilize the AFM coupling but are not expected to have a large effect on the average measured magnetic moment.
\end{abstract}

DOI: 10.1103/PhysRevB.98.024418

\section{INTRODUCTION}

Steels are ubiquitous in technological applications due to the abundance and low cost of $\mathrm{Fe}$ and its highly desirable mechanical and corrosion properties with alloying additions. Manganese is second only to $\mathrm{C}$ in its importance and use in steels. Historically, $\mathrm{Mn}$ has been included as both a minor and major alloying addition — the former to increase workability by suppressing FeS formation and the latter to increase ductility, through twinning induced plasticity, in the fairly recently developed, so-called TWIP steels. The state-of-the art theoretical description of $\mathrm{Mn}$ in Fe has a large impact on our understanding of phenomena such as solute clustering [1] and vacancy-solute clustering [2], which compromise the structural integrity of the steels during operation. The former occurs in the ferritic phase of duplex steels as a result of thermal aging (573-773 K for $>1000 \mathrm{~h})[3-5]$ and in low-alloy steels [6-8] resulting from long-term ( $>1$ year) elevated temperature $(\sim 550 \mathrm{~K})$; the latter occurs due to neutron irradiation damage, which is of interest to life extension of nuclear fission reactors and for fast neutron damage of steels to be used in future fusion reactors. It is therefore important for atomic scale processes such as binding, substitution, and migration to be understood at a fundamental level, to be used in high-order methods and analyses [9-13] to model these phenomena in industrial settings.

Mn has long been regarded as one of the most troublesome transition metals in terms of predicting its magnetic behavior and electronic interaction with other elements [14]. In its elemental ground state, it adopts a 58-atom cubic unit cell of space group $I \overline{4} 3 m$, and exhibits a noncollinear (NC) magnetic structure, whereby atoms located in $2 a, 8 c$, and two $24 g$ sites (in Wyckoff notation) exhibit different spin vectors $[15,16]$.

*Corresponding author: daniel.king@imperial.ac.uk
This magnetic structure undergoes a transition to paramagnetic (PM) structure at the Néel temperature of $\sim 95 \mathrm{~K}$ [17]. In contrast, $\mathrm{Fe}$ is one of the most well studied transition metals [18-20]. In its ground state, Fe adopts a ferromagnetic (FM) bcc structure [21], which it maintains until its Curie temperature at $\sim 1043 \mathrm{~K}$ [22].

With minor alloying additions of $\mathrm{Mn}$ to $\mathrm{Fe}$ the crystal structure will remain bcc as a solid solution. The solubility limit is reached at $\sim 3$ at.\% at $\sim 600 \mathrm{~K}$ [23], at equilibrium; however, increased additions are reported to be stabilized using cold working [24]. The magnetic structure of Fe-Mn alloys is highly variable and is thought to be affected by many different factors, namely, the Mn concentration [25], the local atomic environment [26], lattice parameter [14], and temperature [24]. This behavior is observed to a much lesser extent for $\mathrm{Fe}-\mathrm{Cu}$, $\mathrm{Fe}-\mathrm{Ni}$, and $\mathrm{Fe}-\mathrm{Si}$ solid solutions [27]. It is therefore no surprise that discrepancies exist between experimental observations and theoretical frameworks. In the current study, we use density functional theory (DFT) to study the effect of concentration, local environment, strain, and point defects on the magnetic moment and stability of $\mathrm{Mn}$ in $\alpha$-Fe.

\section{METHODOLOGY}

A plane-wave density functional theory method was used, as implemented in the Vienna Ab Initio Simulation Package (VASP) [28]. The Perdew-Burke-Ernzerhof (PBE) exchangecorrelation functional [29], based on the generalized gradient approximation (GGA), is used with the projector augmented wave (PAW) method [30]. Pseudopotentials provided with the VASP distribution were used, where 14 electrons $\left(3 p^{6} 3 d^{7} 4 s^{1}\right)$ for $\mathrm{Fe}$ and 13 electrons $\left(3 p^{6} 3 d^{5} 4 s^{2}\right)$ for $\mathrm{Mn}$ are considered as valence electrons.

The $k$ points, energy cutoff, and lattice parameters of the perfect lattice of both elements $(\alpha-\mathrm{Fe}$ and $\alpha-\mathrm{Mn})$ were 
converged. It was determined that a real-space $k$-point density of $0.03 \AA^{-3}$ and energy cutoff of $500 \mathrm{eV}$ provided accurate results and was kept consistent for all calculations (resulting in $6 \times 6 \times 6,4 \times 4 \times 4$, and $2 \times 2 \times 2 k$-point grids for the 54-, 128-, and 250-atom supercells). Constant pressure and full relaxations with Methfessel-Paxton [31] smearing (with a width of $0.1 \mathrm{eV}$ ) were used to obtain the ground-state configurations. The tetrahedron smearing method with Blöchl corrections [32] was used on fixed dimension/volume calculations to generate the electronic density of states (DOS). Spin polarization effects were also included; noncollinear and collinear theory was used for the $\alpha-\mathrm{Mn}$ structure. The electronic and ionic energy relaxation convergences were $10^{-6}$ and $10^{-4} \mathrm{eV}$, respectively, for all calculations, and all forces on atoms were converged to

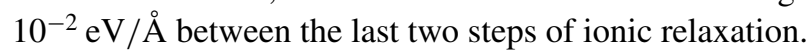

Supercells of $54(3 \times 3 \times 3), 128(4 \times 4 \times 4)$, and $250(5 \times 5 \times 5)$ $\mathrm{Fe}$ atoms were relaxed at constant pressure. To understand the behavior of a dilute concentration of $\mathrm{Mn}$ in the $\alpha$-Fe lattice, a single $\mathrm{Mn}$ was substituted for an $\mathrm{Fe}$ atom in each of the supercell sizes. For higher concentrations of $\mathrm{Mn}$ and $\mathrm{Mn}-\mathrm{Mn}$ interactions within the Fe matrix, a Mn content of 4.69 at.\% was achieved by pseudorandomly selecting six lattice sites for Mn atom substitution in the 128-atom supercell. As this method is stochastic in nature, this was repeated to produce 20 unique supercells (120 Mn atoms in total) to achieve a good statistical understanding of the system. Cohesive, binding, substitutional, vacancy, and interstitial formation energies were calculated following standard procedures, outlined in the Appendix.

Linear elastic theory, as implemented within the ANETO framework [33] for calculating the dipole tensor using the strain method $[34,35]$, was used to calculate the relaxation volume and interaction energies for $\mathrm{Mn}$ in bcc $(\alpha) \mathrm{Fe}$ in the 54-, 128-, and 250-atom supercells.

\section{RESULTS AND DISCUSSION}

\section{A. Pure Mn and Fe}

Table I reports the calculated values of cohesive enthalpies $\left(E_{c}\right)$ and lattice parameters $\left(a_{0}\right)$, presented for the ground-state $(\alpha)$, cubic $(\beta)$, fcc $(\gamma)$, and bcc $(\delta)$ Mn structures. Values for $\alpha$-Fe are also provided, together with past literature values for $\mathrm{Mn}$ and $\mathrm{Fe}$ structures for comparison. The following magnetic structures within $\alpha$-Mn were considered: Nonmagnetic (NM), collinear, antiferromagnetic (AFM), and NC AFM.

In accordance with past literature, and the observed equilibrium behavior of pure $\mathrm{Mn}$, the $\alpha$-Mn phase is the most energetically favorable at $0 \mathrm{~K}[42]$. This is then followed by the $\beta, \gamma$, and $\delta$ phases, which are temperature stabilized. The different known magnetic arrangements of $\alpha$-Mn were simulated; in the NC case, the lowest-energy structure displayed a similar magnetic configuration to the collinear AFM case as found in past literature $[14,16]$. The difference in $E_{c}$ and $a_{0}$ between the NC AFM and collinear AFM $\alpha$-Mn structures is attributed to the spin-orbit interaction $[43,44]$, i.e., the relativistic effect of the magnetization direction to the crystal lattice [45], which was considered in the NC calculation. The magnetic moments of each atomic site can be found in Table III (Appendix). The overestimation of the cohesive enthalpies compared to
TABLE I. Cohesive energy $\left(E_{c}\right)$ and lattice parameter $\left(a_{0}\right)$ of structural and magnetic allotropes of pure $\mathrm{Mn}$ and $\alpha-\mathrm{Fe}$.

\begin{tabular}{|c|c|c|c|c|c|}
\hline \multirow{2}{*}{$\begin{array}{l}\text { Crystal } \\
\text { structure }\end{array}$} & \multirow{2}{*}{$\begin{array}{l}\text { Magnetic } \\
\text { structure }\end{array}$} & \multicolumn{2}{|c|}{$E_{c}(\mathrm{eV} /$ atom $)$} & \multicolumn{2}{|c|}{$a_{0}(\AA)$} \\
\hline & & Current & Literature & Current & Literature \\
\hline \multirow[t]{3}{*}{$\alpha-\mathrm{Mn}$} & NC AFM & 3.87 & & 8.61 & $8.61^{\mathrm{a}}, 8.91^{\mathrm{b}}$ \\
\hline & AFM & 3.86 & & 8.64 & \\
\hline & NM & 3.82 & & 8.56 & \\
\hline$\beta-\mathrm{Mn}$ & AFM & 3.79 & & 6.32 & $6.32^{\mathrm{c}}$ \\
\hline$\gamma-\mathrm{Mn}$ & NM & 3.76 & $3.73^{\mathrm{d}}, 2.92^{\mathrm{e}}$ & 3.50 & $3.49^{f}$ \\
\hline$\delta-\mathrm{Mn}$ & FM & 3.69 & & 2.79 & $2.78^{\mathrm{g}}$ \\
\hline$\alpha-\mathrm{Fe}$ & FM & 4.88 & $4.78^{\mathrm{d}}, 4.28^{\mathrm{e}}$ & 2.83 & $2.86^{\mathrm{e}}$ \\
\hline
\end{tabular}

${ }^{\text {a Reference [14], theoretical. }}$

${ }^{\mathrm{b}}$ Reference [40], experimental.

${ }^{\mathrm{c}}$ Reference [41], experimental.

${ }^{\mathrm{d}}$ Reference [36], theoretical.

${ }^{\text {e}}$ Reference [39], experimental.

${ }^{\mathrm{f}}$ Reference [37], theoretical.

${ }^{\mathrm{g}}$ Reference [38], theoretical.

the experimental values is due to the approximation of the exchange-correlation functional $[36,46]$.

\section{B. Mn in $\alpha-\mathrm{Fe}$}

\section{Magnetic moment and stability}

After gaining confidence in the theoretical representation of pure $\mathrm{Fe}$ and $\mathrm{Mn}$, we move to the case in which a single $\mathrm{Mn}$ solute atom is placed in the $\alpha$-Fe matrix. The substitutional energy $\left(E_{\text {sub }}\right)$ provides an indication of the stability of $\mathrm{Mn}$ in the bcc FM Fe lattice. This value should converge with system size. Past literature has utilized 54- and 128-atom supercells where it was concluded that a convergence of $\pm 0.05 \mathrm{eV}$ was achieved with the 128-atom supercell [47]. However, there is little agreement between the magnetic moment of $\mathrm{Mn}$ and substitutional energy among theoretical reports in the literature [27,47]. In the publication by Olsson et al. [27], it was shown that the substitutional energy can vary $\pm 0.3 \mathrm{eV}$ between the ultrasoft pseudopotential (USPP) [48] and PAW methods and that there is a large discrepancy between the magnetic moments. This was attributed to the inability of the USPP method to correctly represent the semicore $d$ electrons. In this section we investigate the effect of the magnetic moment of $\mathrm{Mn}$ on $E_{\text {sub. }}$. It is known that the magnetic moment of $\mathrm{Mn}$ is particularly difficult to converge in $\alpha-\mathrm{Fe}$ as a shallow energy landscape exists between local FM and AFM states [49] (where all magnetic states are henceforth described, locally, in reference to the Fe matrix). Within this study it was found that the AFM state of Mn is the ground state in $\alpha$-Fe and that it is also possible to model the FM state in 128- and 250-atom supercells, provided a sufficiently high initial magnetic moment is set prior to energy minimization; see Table II. The average magnetic moment of Fe remained relatively consistent at 2.26(1) $\mu_{\mathrm{B}}$ for all calculations within this section.

These results demonstrate that it is possible to converge to both the FM and AFM state of Mn when its initial magnetic moment is 4 or $\leqslant 2$, respectively, for supercell sizes $\geqslant 128$ atoms. The corresponding substitutional energies highlight 
TABLE II. Influence of initial spin on final spin state of $\mathrm{Mn}$ (relative to $\mathrm{Fe}$ ) and the corresponding substitutional energies $\left(E_{\mathrm{sub}}\right)$.

\begin{tabular}{|c|c|c|c|c|c|}
\hline $\begin{array}{l}\text { Supercell } \\
\text { dimensions }\end{array}$ & $\begin{array}{c}\mathrm{Mn} \\
\text { concentration } \\
(\text { at. } \%)\end{array}$ & $\begin{array}{c}\text { Initial } \\
\text { spin } \mathrm{Mn} \\
\left(\mu_{\mathrm{B}}\right)\end{array}$ & $\begin{array}{c}\text { Final } \\
\text { spin } \mathrm{Mn} \\
\left(\mu_{\mathrm{B}}\right)\end{array}$ & $\begin{array}{c}\text { Final } \\
\text { spin state } \\
\text { Mn }\end{array}$ & $E_{\text {sub }}(\mathrm{eV})$ \\
\hline $3 \times 3 \times 3$ & 1.85 & $\begin{array}{r}-2.00 \\
0.00 \\
2.00 \\
4.00\end{array}$ & -1.89 & AFM & 0.20 \\
\hline $4 \times 4 \times 4$ & 0.78 & $\begin{array}{r}-2.00 \\
0.00 \\
2.00 \\
4.00\end{array}$ & -1.98 & AFM & 0.19 \\
\hline $5 \times 5 \times 5$ & 0.40 & $\begin{array}{r}-2.00 \\
0.00 \\
2.00 \\
4.00\end{array}$ & -2.08 & AFM & 0.20 \\
\hline
\end{tabular}

the importance of the resultant magnetic moment of $\mathrm{Mn}$ to its stability in the $\alpha$-Fe matrix. When it is in the FM state the $E_{\text {sub }}$ becomes less favorable by 0.10 and $0.05 \mathrm{eV}$ for the $4 \times 4 \times 4$ and $5 \times 5 \times 5$ supercell, respectively. Since it is predicted that the AFM state is the more stable of the two, it is recommended that it is used as the reference state for the calculation of binding energy calculations.

When assessing the local density of states (LDOS) of Mn in $\alpha$-Fe, the FM and AFM spin states are distinct; see Fig. 1. The difference in states is observed as the shift in the antibonding peak of the majority spin channel, from above the Fermi level for the AFM, to below the Fermi level in the FM magnetic coupling of $\mathrm{Mn}$. The occupation of the antibonding peak explains the relative stability difference between the two states [50].

\section{Calculated vs experimental magnetic moment}

In this section we compare the DFT calculated magnetic moments in the current study to the past experimental studies that used diffuse neutron scattering. For the former, the Mn concentration was varied by using the three different supercell sizes; 250, 128, and 54 atoms correspond to $0.40,0.78$, and 1.85 at. \%, respectively. A higher concentration of 4.69 at.\% (above the expected solubility limit) was obtained by randomly populating the 128-atom supercell with six Mn atoms, repeated in 20 supercells; the average of all the magnetic moments was taken and the standard error is plotted as error bars.

Figure 2 shows that there is little agreement among the experimental data and that the ground-state theoretical values, obtained in this work, overpredict the magnitude of the magnetic moment below 5 at.\%. However, it should be emphasized that the solubility of $\mathrm{Mn}$ in $\alpha-\mathrm{Fe}$ is at maximum $\sim 3$ at. $\%$ at $600 \mathrm{~K}$ [23]. In the studies that surpass this solubility, Nakai and Kunitomi [24] reportedly stabilize the $\alpha$ phase by cold working and the study by Radhakrishna and Livet [26] does not provide such information. Formation of secondary phases such as $\gamma$-(Mn,Fe) is likely to occur at equilibrium for $\mathrm{Mn}$ concentrations $>3$ at. $\%$. Nevertheless, the results in the current

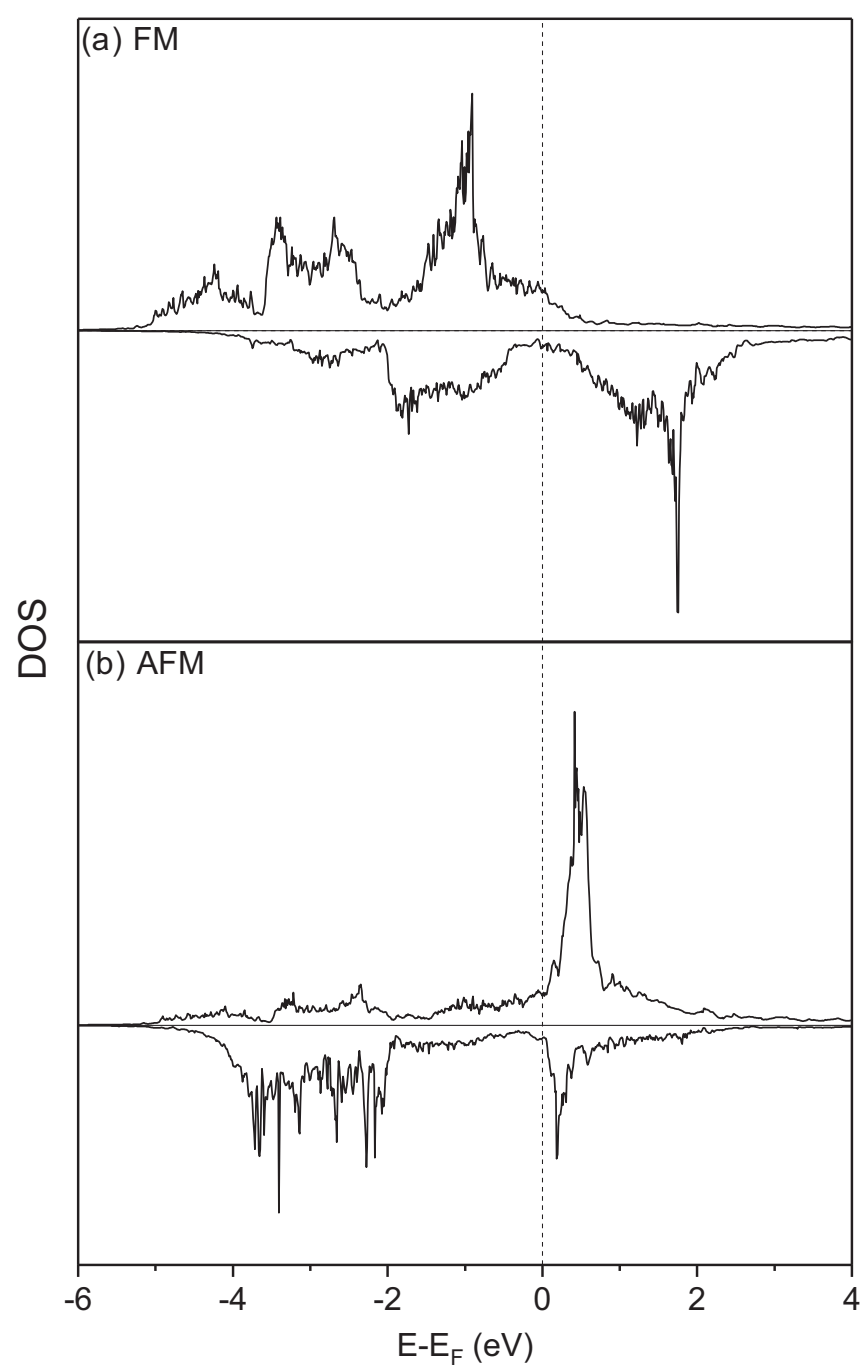

FIG. 1. Local density of states of the $d$ band of (a) FM and (b) AFM spin coupling in $\alpha$-Fe for a 128 -atom supercell.

study are in better agreement with experimental findings for $\mathrm{Mn}$ in excess concentrations.

In the following sections, the possible factors that lead to the discrepancies between experiment and theoretical calculations and how these differences can be captured through a mechanistic understanding of the Fe-Mn system are explored.

\section{Atomic relaxations}

By taking the difference between the atomic coordinates of the relaxed and unrelaxed supercells, the vectors corresponding to the atomic relaxations upon substitution of Fe with Mn can be calculated. Figure 3 provides an illustrative representation of the vectors $(\times 100)$ in a 250 -atom supercell. Although these relaxations are small $\left(\sim 10^{-2} \AA\right)$ they are markedly different between FM and AFM states. For the AFM relaxation, the first nearest neighbor (1st $\mathrm{nn}$ ) Fe was repelled by the $\mathrm{Mn}$ atom. The repulsion propagated along $\langle 111\rangle$ over relatively long distances $(\sim 10 \AA)$, while Fe atoms along $\langle 100\rangle$ and $\langle 201\rangle$ from the Mn experience a shorter-range attraction $(\sim 5 \AA)$. In the FM case, only displacements of Fe towards Mn are observed 


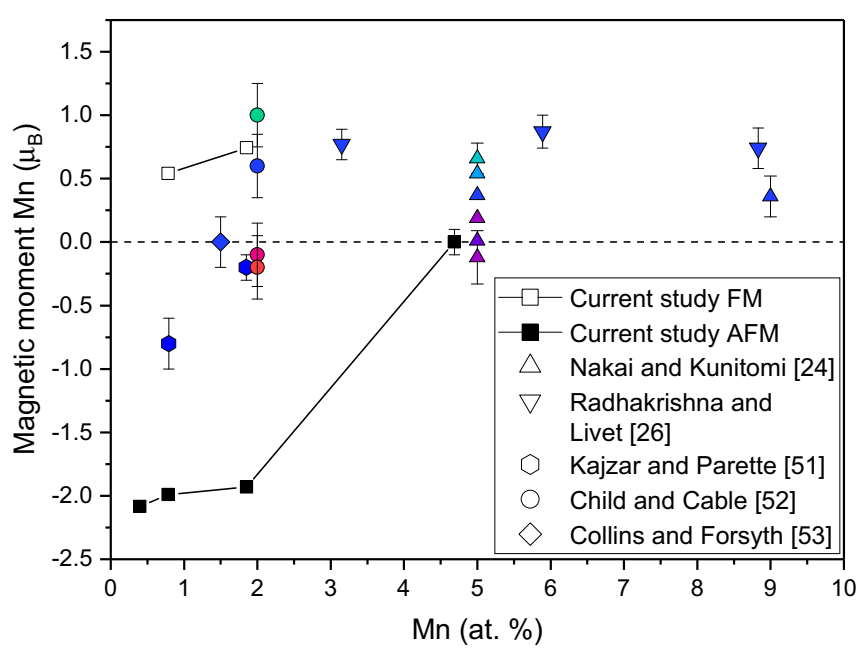

FIG. 2. Magnetic moment of $\mathrm{Mn}$ in $\alpha$-Fe as calculated using DFT in the current study for AFM Mn (closed squares) and FM Mn (open squares) compared to the experimental magnetic moments using diffuse neutron scattering, as measured by Nakai and Kunitomi [24] (colored triangles), Radhakrishna and Livet [26] (colored inverted triangles), Kajzar and Parette [51] (colored hexagons), Child and Cable [52] (colored circles), and Collins and Forsyth [53] (colored diamonds) where the colors represent the temperature of the sample upon measurement.

(compressive), with the largest magnitude of $1.3 \times 10^{-2} \AA$ in the 1 st nn Fe.

For the smaller supercells (54 and 128 atoms) the long-range movement is more constrained and may explain the slight variance in magnetic moment and absence of FM moment for the 54-atom case. Figure 4 shows the elastic self-interaction energy [54,55]. It can be seen that the supercells are well converged by 128 atoms.

From linear elastic theory, the relaxation volumes of FM and AFM Mn are calculated for the different supercell sizes; see Fig. 5. The volume change due to the tensile relaxations of the AFM Mn and compressive relaxations of the FM Mn can be seen clearly. The effect of the FM Mn on the Fe lattice is much more localized than the AFM, where the former has self-interaction energies three orders of magnitude smaller than the latter. These results suggest that the increased stability of

AFM

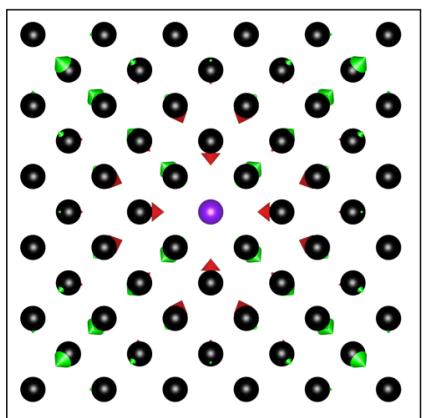

FIG. 3. Atomic relaxation vectors $\times 100$ in compression (red) and tension (green) of $\mathrm{Fe}$ atoms around a Mn substitution (purple) for the AFM and FM magnetic moment of $\mathrm{Mn}$ in the 250-atom supercell.

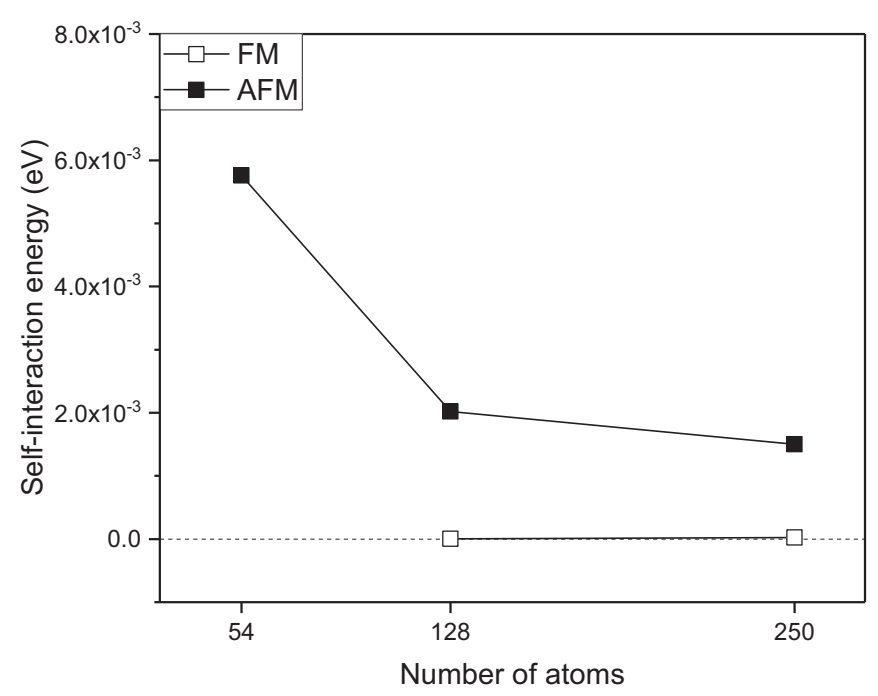

FIG. 4. Self-interaction energy of the substitutional Mn defect in $\alpha$-Fe when in the FM and AFM magnetic state.

the AFM state over the FM state of Mn is due to electronic rather than elastic effects.

\section{Mn concentration}

The interaction between neighboring Mn species in $\alpha$-Fe is not well understood. Past theoretical studies that report the binding energies between two Mn atoms are not in accord $[27,47,56]$. Within this study, a stochastic behavior was found in the resultant magnetic moments of the Mn. When two Mn atoms were placed in the 54-, 128-, and 250-atom supercells, in different nn positions, each adopted FM or AFM moments with varying magnitudes and no clear trend (reported in Fig. 13 of the Appendix). The binding energies between the two Mn atoms varied by $\pm 0.05 \mathrm{eV}$ between supercells and did not correlate with their magnetic moment. Figure 6 reports the binding energies calculated for the 250-atom supercell, and

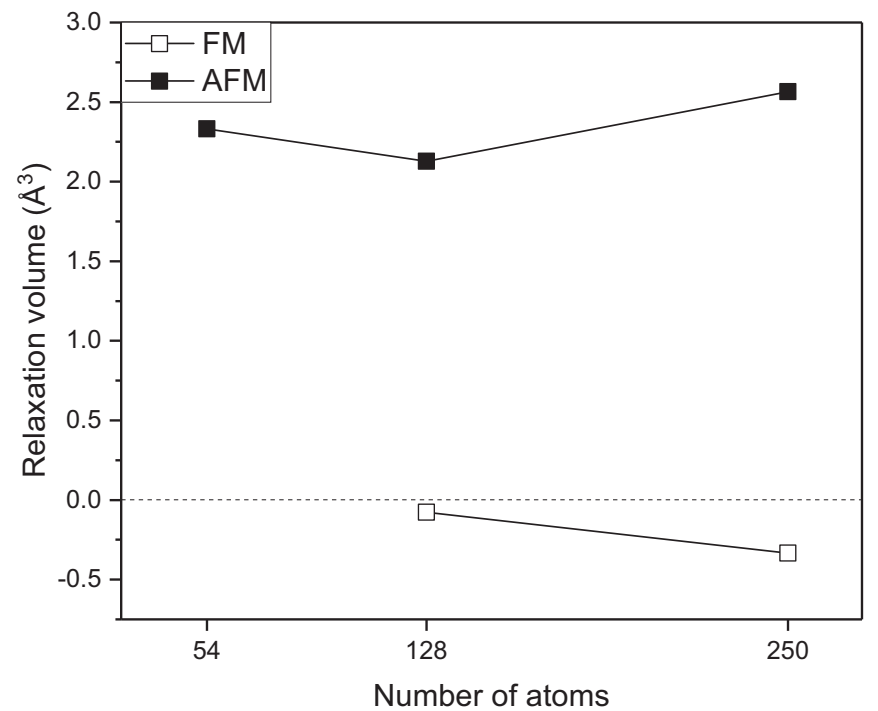

FIG. 5. Relaxation volume of the substitutional Mn defect in $\alpha$-Fe when in the FM and AFM magnetic state. 


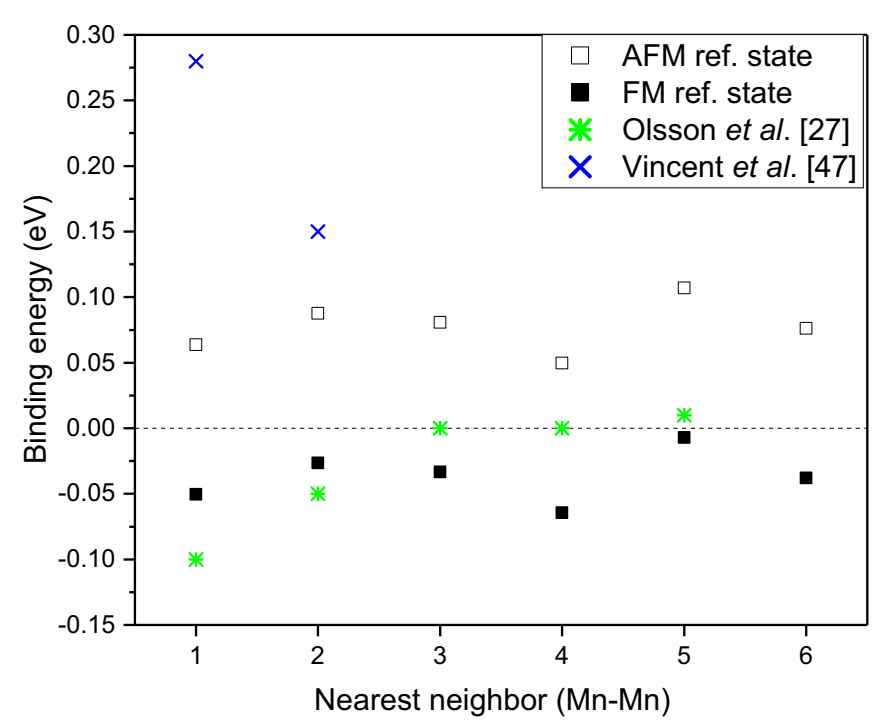

FIG. 6. Mn-Mn nn binding energy for the 250-atom supercells using the AFM (open square) and FM (closed square) reference state. Comparison to the work of Olsson et al. [27] (green star) and Vincent et al. [47] (blue cross) is included.

provides a comparison with past literature. The calculation of the binding energy was done using the AFM and FM reference state, which results in a significant difference $(0.14 \mathrm{eV})$ in energy.

When comparing to the work of Olsson et al. [27], the average binding energies calculated from the FM Mn in the $\alpha$-Fe reference state are somewhat in agreement. However, the FM state is metastable; therefore the AFM state should be taken as reference. This offset does not explain the discrepancy with respect to the work of Vincent et al. [47]. It is more likely that the difference stems from the use of USPPs and other theoretical parameters.

When considering the difference in atomic relaxations for AFM and FM isolated $\mathrm{Mn}$ atoms, it is perhaps no surprise that there is a large variation in magnetic moment between nearest neighbor Mn with varying supercell size. If Mn does indeed prefer to exhibit AFM behavior, then a more complex atomic relaxation is required. However, at certain geometries of nearest neighbor $\mathrm{Mn}$, and without sufficient long-range movement allowed, it will be more favorable for AFM and FM Mn atoms to coexist.

A more concentrated system, in which six Mn atoms are randomly distributed on a 128 -atom bcc lattice replacing Fe, corresponding to a $\mathrm{Mn}$ concentration of 4.69 at.\%, is now considered. Previous theoretical work on concentrated Fe-Mn systems conclude that there is a uniform switch from the AFM to FM moment in Mn between 2 and 3 at.\% [57,58]. In the current study, without sufficient statistical precision, this conclusion could mistakenly be made; of the 20 supercells studied, $15 \%$ displayed exclusively FM moments for Mn. However, the magnetic moments of Mn within the other $85 \%$ of the supercells were a mix of AFM and FM where the average moments across all supercells range from $-0.8 \mu_{\mathrm{B}}$ to $0.9 \mu_{\mathrm{B}}$. Figure 7 shows the distribution of the magnetic moments of each $\mathrm{Mn}$ atom plotted against supercell energy. When taking the average of all $120 \mathrm{Mn}$ atoms simulated, the magnetic

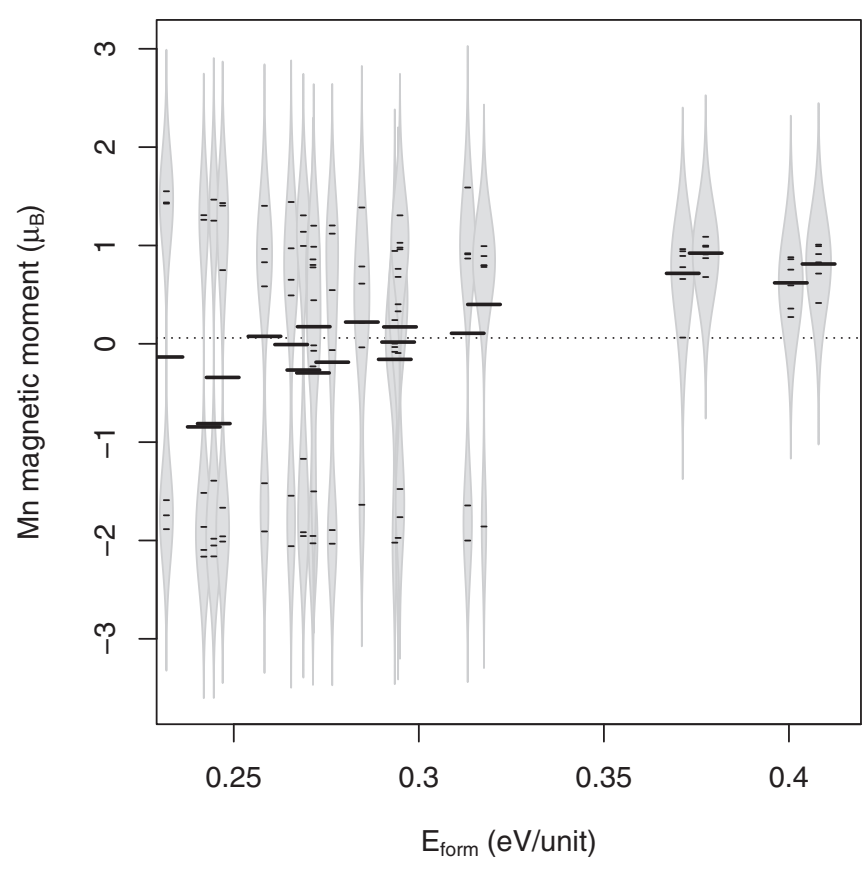

FIG. 7. Magnetic moment of each Mn atom (small dashes), across the 20 supercells (gray shading), versus the enthalpy of formation per unit ( $3 \mathrm{Mn}: 51 \mathrm{Fe})$. The average magnetic moment per supercell and mean magnetic moment across all supercells is denoted by the large dashes and dotted line, respectively.

moment equates to $0.06 \mu_{\mathrm{B}}$ (dotted line). The enthalpy of formation $\left(E_{\text {form }}\right)$ of the system displays a general trend whereby the systems with lower average magnetic moment (large dashes) are more energetically favorable. No correlation was found (correlation coefficient $=0.013$ ) between the nearest neighbor species and the resultant magnetic moment of Mn, which vary between $-2.2 \mu_{\mathrm{B}}$ and $1.1 \mu_{\mathrm{B}}$; see Fig. 14 .

The environmental effect that is thought to influence the magnetic moment of Mn [26] cannot be predicted by the nearest neighbor species or concentration of $\mathrm{Mn}$ in $\alpha-\mathrm{Fe}$. Further, the presence of other Mn species does not stabilize a particular spin state. Rather, a more complex effect, likely due to the relaxation vectors around each $\mathrm{Mn}$, is observed, resulting in an average moment close to zero (and median of $\sim 0.70 \mu_{\mathrm{B}}$ ). The interaction volumes used in diffuse neutron scattering vary greatly with experimental parameters [59]; it is expected that these volumes are of the $\mu \mathrm{m}$ length scale (although not explicitly stated) in references from Sec. III B 2. Therefore, the overall average magnetic moment is an important result for comparison to the literature.

\section{Effect of strain}

In the past, it has been demonstrated that the lattice parameter of $\alpha-\mathrm{Mn}$ has a large effect on its magnetic structure [14]. To date this effect has not been investigated in the Fe-Mn system; however, pioneering work by Cable and Tsunoda has explored this effect in Ni- and Co-Mn alloys [60]. Two methods of straining the environment around the $\mathrm{Mn}$ atom were used in this study, both using 128-atom supercells: applying tension and compression (1) hydrostatically and fixing the volume and shape of the supercell while allowing internal relaxation of the 


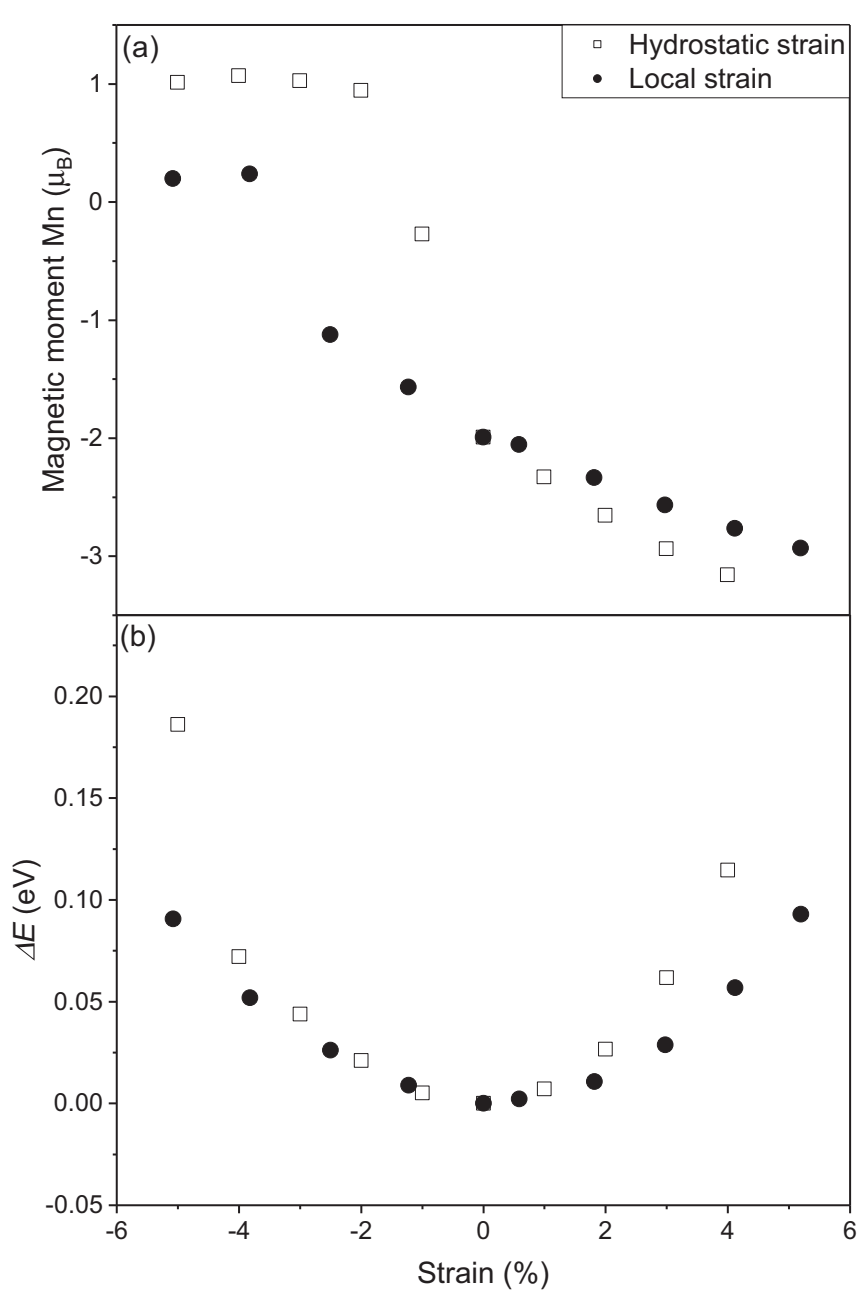

FIG. 8. Magnetic moment of Mn (a) and difference in internal energy of strained and ground-state supercell, $\Delta E$, normalized by the number of strained atoms (b) with a comparison between hydrostatic strain (open squares) and local 1st nn strain (closed circles).

atoms, and (2) locally in the 1st nn Fe, fixing supercell shape and atomic positions of atoms. The latter was performed to investigate the influence of only the $1 \mathrm{st} \mathrm{nn}$.

At the dilute limit, the effect of strain on the magnetic moment of $\mathrm{Mn}$ is clear; compression (negative strain) induces the FM moment and tension induces the AFM moment; see Fig. 8(a). The results from the hydrostatic method show a transition from AFM to FM moment between $0 \%$ and $-2 \%$ strain. For the locally strained case, the transition occurs at higher compressive strains and to a lesser degree. This discrepancy is considered to be the result of the influence of the neighboring atoms, other than the 1st nn, which remain fixed. The difference in internal energy is calculated as

$$
\Delta E=\frac{E_{\text {strained }}-E_{g s}}{n_{d}}
$$

where $E_{\text {strained }}$ and $E_{g s}$ are the total internal energy of the strained and ground-state supercell, respectively, normalized to the number of displaced atoms $\left(n_{d}\right)$. The results follow the expected parabolic distribution where deviation from the ground-state configuration leads to less favorable energies [Fig. 8(b)].

These results suggest that the disagreement between the observed experimental magnetic moment of Mn is likely due to the different conditions of the samples. Nonequilibrium features such as increased defect concentrations and residual stress, due to quenching or cold working, are expected to have a large impact on the measured magnetic moment of $\mathrm{Mn}$ in $\mathrm{Fe}$. A lattice parameter change of $0.03 \AA$ equates to a strain of $1 \%$. Experimentally, an increase in temperature of $\sim 250 \mathrm{~K}$ leads to a lattice parameter increase of $0.01 \AA$ for $\alpha$-Fe [61]. However, as the alloy approaches its Curie temperature a rapid drop in net magnetic moment will occur [62]. Neglecting the phonon interactions and other temperature effects, it may be possible to observe both AFM and FM average moments in samples with the same concentration of $\mathrm{Mn}$, as seen by Child and Cable [52] and Nakai and Kunitomi [24], providing the temperature is varied sufficiently and is below the Curie temperature or the sample is under sufficient strain. However, caution must be used when making direct comparisons to experiment. This is because the approximation of the exchange-correlation functional used by DFT introduces discrepancies to both the lattice parameter and the magnitude of the magnetic moment [63]. Therefore, this result should be taken qualitatively and an experimental relation between strain and magnetic moment is required to establish a quantitative relationship.

\section{Effect of vacancies}

Vacancies are ubiquitous in crystalline systems and vary in concentration depending on the condition and processing of the sample [64]. Vacancy-solute clusters are a documented phenomenon $[65,66]$ and the binding of solute atoms to vacancies in $\alpha$-Fe is important for understanding solute partitioning, diffusivity, and precipitation [67-69]. In Figs. 9(a)-9(c), the resultant magnetic moment, binding energy, and vacancy formation energy of Mn substitution are shown as a function of distance from a vacancy from 1st to 6th $\mathrm{nn}$.

The magnitudes and trend of the binding energies are in accordance with past theoretical findings $[27,47,70]$. It is predicted that it is favorable for $\mathrm{Mn}$ to bind to an Fe vacancy, with a relatively long-range attraction compared to other $3 d$ transitional metals $[27,71,72]$. There is a clear stabilization of the AFM moment of Mn when in the 1st nn position and the binding energy between $\mathrm{Mn}$ and vacancy follows a similar trend of the magnetic moment for all supercell sizes. This behavior is different from many of the other transition metals, which show little variance in magnetic moment with vacancy position [27]. The vacancy formation enthalpies $\left(E_{\mathrm{vac}}\right)$ follow the same trends seen in the magnetic moment and binding energies. Compared to pure $\mathrm{Fe}$, there is a significant lowering in $E_{\mathrm{vac}}$ in the 1st and 2nd nn to the Mn. Again, it must be emphasized that, when calculating the binding energy, the use of the metastable FM Mn reference state in $\alpha$-Fe leads to a difference in binding energy of $0.06 \mathrm{eV}$ (more favorable) to the ground-state AFM reference state.

The nonlinear trend in binding energies is interesting, especially because it is expected that the binding energy should tend to zero with increasing distance. Here, it is found that the Friedel-like perturbations of the Fe around the vacancy 


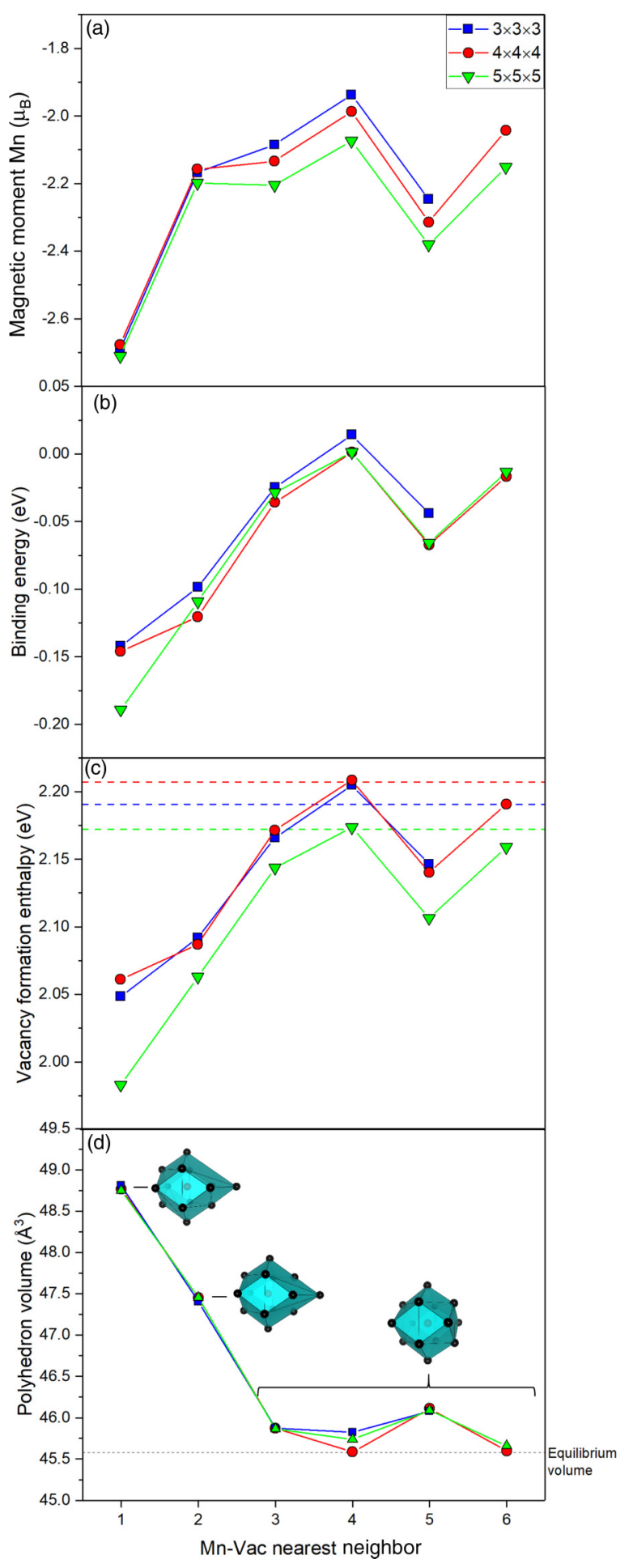

FIG. 9. (a) Mn local magnetic moment, (b) Mn-vac binding energy, and (c) vacancy formation enthalpy in the $\alpha$-Fe matrix for 1 st to 6 th $\mathrm{nn}$ for a $3 \times 3 \times 3$ (blue square), $4 \times 4 \times 4$ (red circle), and $5 \times 5 \times 5$ (green triangle) dimension supercell; dashed lines with the same colors in (c) represent the vacancy formation enthalpies of pure Fe. (d) The volumes of the polyhedron created by the 1 st and 2 nd $\mathrm{nn}$ around the Mn solute atom. When the Mn was in 1st and 2nd nn proximity to the vacancy the polyhedron was extended to the $3 \mathrm{rd} \mathrm{nn}$ in that direction (pictorial representations). The equilibrium volume for a single $\mathrm{Mn}$ in the $\alpha$-Fe matrix is provided for reference (dashed line).

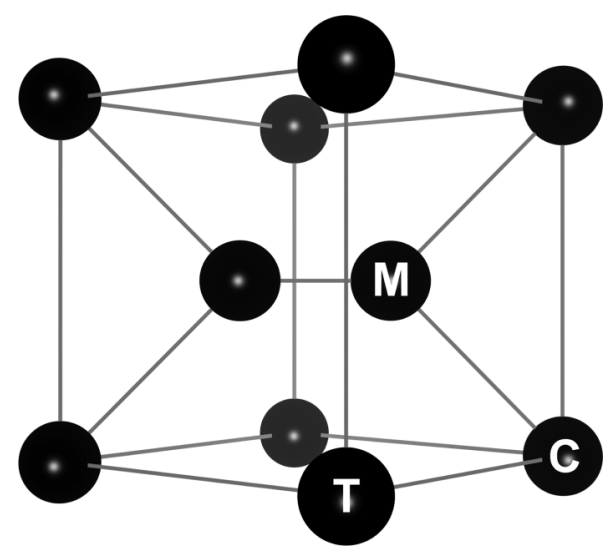

FIG. 10. $\langle 110\rangle$ interstitial dumbbell configuration in $\alpha$-Fe. Labels $\mathrm{M}$ (mixed), C (compressed), and $\mathrm{T}$ (tensile) denote the position of the Mn substitution with respect to a dumbbell.

dominate over those around the Mn substitution. By assessing the volume of the polyhedron created by the 1 st and $2 \mathrm{nd} n \mathrm{n}$ around the $\mathrm{Mn}$ atom, a negative correlation to the binding energies and magnetic moment is found; see Fig. 9(d). When $\mathrm{Mn}$ is in the 1st and 2nd nn (to the vacancy) the polyhedron is extended to the 3rd nn (from Mn) in the direction of the absent site. These findings follow the trend identified within this study; i.e., the FM moment is stabilized by compressive and AFM by tensile lattice strain. At equilibrium, without a vacancy, the $2 \mathrm{nd} n$ polyhedron volume containing $\mathrm{Mn}$ is calculated to be $45.60 \AA^{3}$, which corresponds to the groundstate magnetic moment of $-2 \mu_{\mathrm{B}}$. When only using $1 \mathrm{st} n n$ polyhedron volumes no correlation was found.

From an applied perspective the equilibrium vacancy concentration is quite small in $\mathrm{Fe} \leqslant 10^{-6}$ below the melting temperature $[2,73]$. However, when subject to displacive radiation damage these concentrations can become nontrivial. It is possible that the increased stability when $\mathrm{Mn}$ is under local tensile strain, in the presence of a vacancy, is the basis for solute-vacancy stabilization seen experimentally [2] or hypothesized to occur due to nucleation of solutes from defects in reactor pressure vessel steels [6].

\section{Effect of the $\langle 110\rangle$ Fe interstitial}

Unlike the majority of bcc metals, the most stable selfinterstitial configuration in $\alpha$-Fe is the $\langle 110\rangle$ dumbbell [74]. Although Mn has been shown to also have a strong binding to the $\langle 111\rangle \mathrm{Fe}$ dumbbell interstitial, it is expected that the $\langle 110\rangle$ will be the most prevalent interstitial in $\alpha$-Fe [27]. For this reason, only the $\langle 110\rangle$ orientation was considered with $\mathrm{Mn}$ at three positions around the Fe interstitial: mixed (M), compressive (C), and tensile (T); see Fig. 10. Within this study these denotations are arbitrary; however, to remain consistent to past literature where the names are given in reference to their respective Voronoi polyhedra volumes [12], we continue their use. From our current work, it is identified that the 2nd nn displacement has a non-negligible effect on the magnetic moment of Mn (Sec. III B 5); therefore a Voronoi analysis is not expected to correlate with the resultant magnetic moment of $\mathrm{Mn}$. 

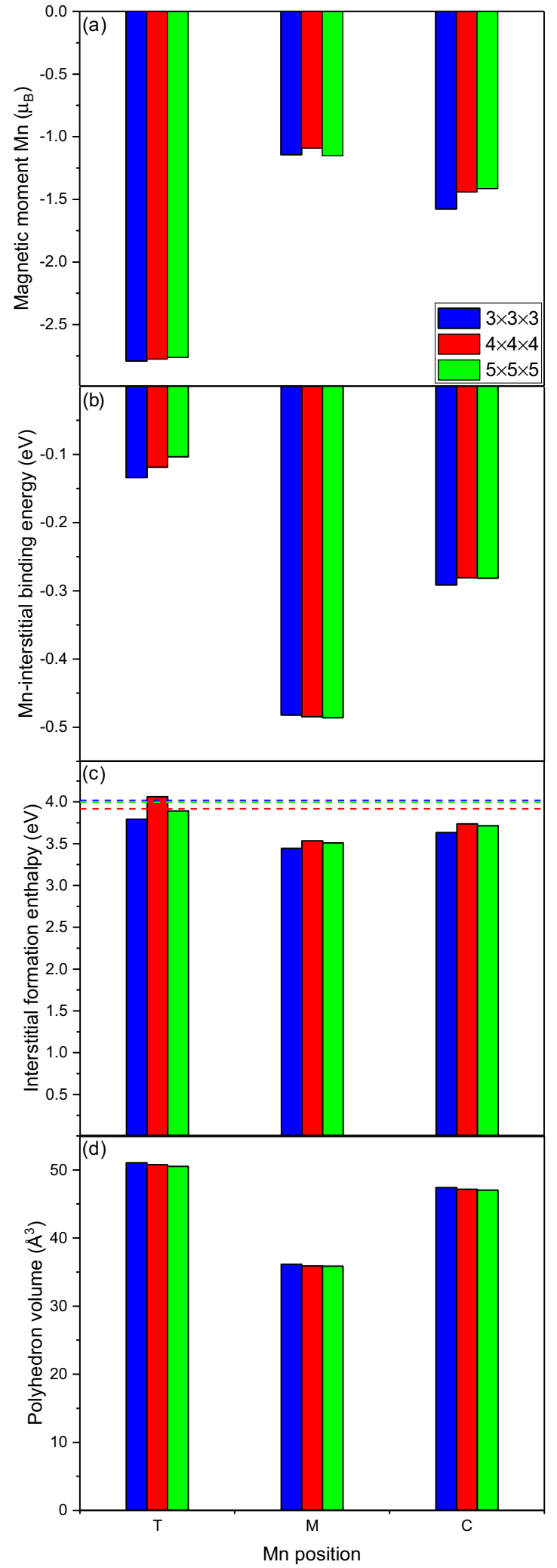

FIG. 11. (a) Mn local magnetic moment, (b) binding energy, and (c) $\langle 110\rangle$ interstitial formation enthalpy in the $\alpha$-Fe matrix for T, M, and $\mathrm{C}$ sites for a $3 \times 3 \times 3$ (blue), $4 \times 4 \times 4$ (red), and $5 \times 5 \times 5$ (green) dimension supercell; dashed lines with the same colors in (c) represent the interstitial formation energies of pure Fe. (d) The volumes of the polyhedron created by the 1 st and 2 nd nn around the Mn solute atom.

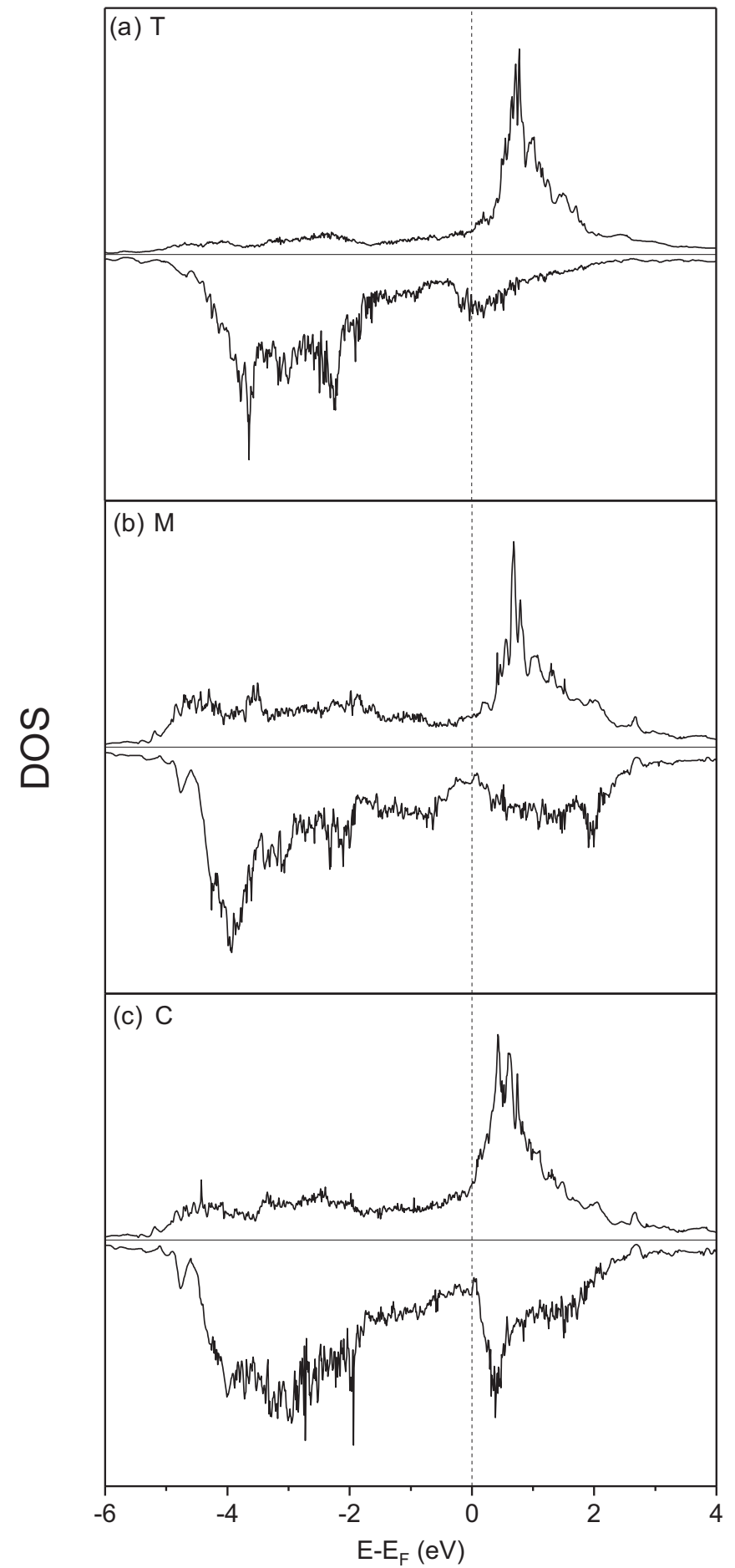

FIG. 12. Local density of states of the combined $s, p$, and $d$ bands of $\mathrm{Mn}$ in the (a) T, (b) $\mathrm{M}$, and (c) $\mathrm{C}$ positions relative to a $\langle 110\rangle$ dumbbell interstitial in $\alpha$-Fe for the 128-atom supercells.

In agreement with past work, the binding energies between $\mathrm{Mn}$ and an $\mathrm{Fe}\langle 110\rangle$ interstitial were favorable in all three sites; see Fig. 11. A difference in binding energy between FM and AFM Mn reference state was found to be 0.08 and $0.06 \mathrm{eV}$ for $4 \times 4 \times 4$ and $5 \times 5 \times 5$ supercells, respectively. Interestingly, the relative magnetic moments do not follow the same trend as seen with the vacancy defects. Analysis of the 2 nd nn 
polyhedra volumes again displays a negative correlation with the magnetic moments, as seen with the vacancies.

In the absence of $\mathrm{Mn}$, the magnetic moment of the two $\mathrm{Fe}$ atoms in the $\langle 110\rangle$ dumbbell configuration exhibits a weak AFM coupling to the matrix $-0.27(2) \mu_{\mathrm{B}}$. Further, the 1 st $\mathrm{nn} \mathrm{Fe}$ atoms to the dumbbell display a significant deviation in magnitude of their magnetic moment from the matrix. The four $\mathrm{Fe}$ atoms in the geometrically equivalent $\mathrm{C}$ and $\mathrm{T}$ sites yield magnetic moments of $\sim 1.72(7)$ and $\sim 2.42(1) \mu_{\mathrm{B}}$, respectively, which vary only slightly with Mn substitution. It is likely that the introduction of a self-interstitial atom sufficiently changes the atomic and magnetic structure so that the magnitudes of the 2 nd nn polyhedron volume vs magnetic moment relationship, determined for the undefective and vacated cases, no longer hold true. To quantify the uncertainty due to the exchange-correlation functional, the Armiento and Mattsson (AM05) implementation of the GGA [75] was applied in the 54-atom supercells of $\mathrm{Fe}$ and with additions of $\mathrm{Fe}$ and $\mathrm{Mn}\langle 110\rangle$ interstitial atoms. The difference in magnetic moment and interstitial formation energy of Fe was small ( $\pm 0.17 \mu_{\mathrm{B}}$ and $\pm 0.07 \mathrm{eV}$, respectively). Little difference in the magnetic moments and binding energies was found for $\mathrm{Mn}\left( \pm 0.28 \mu_{\mathrm{B}}\right.$ and $\pm 0.16 \mathrm{eV}$, respectively). A larger difference in $\mathrm{Mn}$ interstitial formation energy was calculated $( \pm 0.61 \mathrm{eV})$. Again, it is emphasized that a qualitative message should be taken from the analysis within this section; i.e., Mn will adopt an AFM moment when in the area around the site of a self-interstitial defect; further evidenced by their LDOS that display characteristic patterns of an AFM Mn with unfilled antibonding peaks; see Fig. 12.

\section{CONCLUSIONS}

(1) The discrepancy in magnetic moment of $\mathrm{Mn}$ in $\alpha$-Fe, that exists between theory and experiment, is likely due to susceptibility of the magnetic moment to change with strain. Here, it was found that a hydrostatic compressive strain of $-2 \%(0.06 \AA)$ stabilized the FM state of Mn atoms (relative to $\mathrm{Fe}$ ). Strains imposed by quenching, cold working, or thermal expansion are expected to dominate over the effect due to Mn concentration, vacancies, and Fe interstitial atoms. Therefore, it is extremely important to provide experimental details pertaining to the lattice parameter and sample conditions in future assessment of the magnetic moment of $\mathrm{Mn}$ in Fe.

(2) In the dilute case, it is possible to simulate both FM and AFM states of Mn, where the former is metastable, by initializing the spin state on Mn to 4 and $\leqslant 2 \mu_{\mathrm{B}}$, respectively. The relaxations of the Fe atoms around the FM and AFM Mn differ significantly. A long-range tensile relaxation in $\langle 111\rangle$ and compression in $\langle 100\rangle$ and $\langle 201\rangle$ occur for the AFM case and strictly compressive relaxations for the FM. The AFM state is the ground state for dilute Mn defects in $\alpha$-Fe and should be used as a reference state to calculate the binding energies of defect complexes in future work.

(3) At higher Mn concentrations, Mn exhibits AFM and FM spin states in a stochastic manner with an average moment of $\sim 0.06 \mu_{\mathrm{B}}$ and median of $\sim 0.70 \mu_{\mathrm{B}}$. By providing a statistically significant dataset, we show that the previous theory of a uniform switch from AFM to FM between 2 and 3 at.\% $\mathrm{Mn}$ is an artefact of inadequate statistical sampling.
This randomness is likely due to a combination of shallow energy landscape between AFM and FM spin states and the inability for relaxations of the surrounding atoms to occur for specific geometries and concentrations of $\mathrm{Mn}$, as to allow for exclusively one state to exist.

(4) A vacancy and $\langle 110\rangle \mathrm{Fe}$ interstitial were both found to stabilize the AFM moment of Mn. The atomic relaxations due to the defects dominate over those around the Mn substitution. Polyhedron analysis of the atoms within the 2 nd nn to $\mathrm{Mn}$ show a negative correlation between volume and magnetic moment with varying $n$ distance to the vacancy/interstitial. This result suggests that the relationship between magnetic moment and local strain still exists when in the presence of a vacancy or interstitial.

\section{ACKNOWLEDGMENTS}

M.R.W. and D.J.M.K. were funded by EPSRC Grant No. EP/P005101/1. T.M.W. acknowledges support through the CDT in nuclear energy Grant No. EP/L015900/1 and funding from Rolls-Royce Plc. This research was undertaken with the assistance of resources provided by the EPSRC Tier 2 allocation on CSD3 (Skylake and KNL) HPCs, Australian National Computational Infrastructure provided by UNSW (Raijin), and Imperial College Tier 2 computing ( $\mathrm{Cx} 1$ and Cx2). We would also like to thank Dr. G. Malcolm Stocks for this discussion and contribution to the work.

\section{APPENDIX}

\section{Methods}

\section{a. Cohesive enthalpy}

Reference energies of isolated Fe and Mn were obtained by placing one atom of each element in separate supercells of dimensions $12 \times 13 \times 14 \AA$. A non-self-consistent calculation at the $\Gamma$ point was performed without symmetry to determine the anisotropic orbital occupancies of the free atom. The magnetic moments for Fe and $\mathrm{Mn}$ were initialized as 4 and $4.4 \mu_{\mathrm{B}}$, respectively. The occupancies were found as follows:

\begin{tabular}{lc}
\hline Fe & Mn \\
Spin-up channel: $3 p^{3} 3 d^{5} 4 s^{1}$ & Spin-up channel: $3 p^{3} 3 d^{5} 4 s^{1}$ \\
Spin-down channel: $3 p^{3} 3 d^{1} 4 s^{1}$ & Spin-down channel: $3 p^{3} 4 s^{1}$ \\
\hline \hline
\end{tabular}

Subsequent self-consistent energy calculations were performed until an electronic convergence of $10^{-6} \mathrm{eV}$ was achieved. The cohesive enthalpy was then calculated using the following equation:

$$
E_{c}=E^{\text {bulk }}-E^{\text {free }},
$$

where $E^{\text {bulk }}$ and $E^{\text {free }}$ are the internal energy per atom of the element in its bulk and free state, respectively.

\section{b. Formation, substitution, binding energy calculations}

Calculations of the enthalpy of formation were performed as follows:

$$
H_{\mathrm{form}}=E_{\mathrm{Fe}, \mathrm{Mn}}-\left(n_{\mathrm{Fe}} E_{\mathrm{Fe}}^{\mathrm{bulk}}+n_{\mathrm{Mn}} E_{\mathrm{Mn}}^{\mathrm{bulk}}\right),
$$


TABLE III. Magnetic moments of the allotropes of Mn.

\begin{tabular}{lccccc}
\hline \hline \multirow{2}{*}{$\begin{array}{l}\text { Crystal } \\
\text { structure }\end{array}$} & Magnetic & & & \multicolumn{3}{c}{ Spin vector } \\
\cline { 4 - 6 } & structure & Site & $x$ & $y$ & $z$ \\
\hline & & $2 a$ & 0.00 & 0.00 & 2.90 \\
& & $8 c$ & 0.00 & 0.00 & -2.17 \\
$\alpha-\mathrm{Mn}$ & $\mathrm{NC}$ & $24 g$ & 0.00 & 0.00 & 0.45 \\
& & $24 g^{\prime}$ & 0.00 & 0.00 & -0.13 \\
& & $2 a$ & & & 2.85 \\
& & $8 c$ & & & -2.3 \\
& $\mathrm{AFM}$ & $24 \mathrm{~g}$ & & & 1.20 \\
$\beta-\mathrm{Mn}$ & & $24 \mathrm{~g}^{\prime}$ & & & -0.01 \\
& $\mathrm{AFM}$ & $8 c$ & & & -0.12 \\
$\gamma-\mathrm{Mn}$ & & $12 d$ & & & 0.45 \\
$\delta-\mathrm{Mn}$ & $\mathrm{NM}$ & $4 a$ & & & 0.90 \\
$\alpha-\mathrm{Fe}$ & $\mathrm{FM}$ & $2 a$ & & & 2.20 \\
\hline \hline
\end{tabular}

where $E_{\mathrm{Fe}, \mathrm{Mn}}$ is the internal energy of the Fe-Mn alloy and $n$ is the number of atoms of the respective element in the alloy.

The calculations for the substitution energies were done using a similar method; however, $n_{\mathrm{Mn}}=1$. Therefore

$$
E_{\mathrm{sub}}=E_{\mathrm{Fe}, \mathrm{Mn}}-\left(n_{\mathrm{Fe}} E_{\mathrm{Fe}}^{\text {,bulk }}+E_{\mathrm{Mn}}^{\text {bulk }}\right),
$$

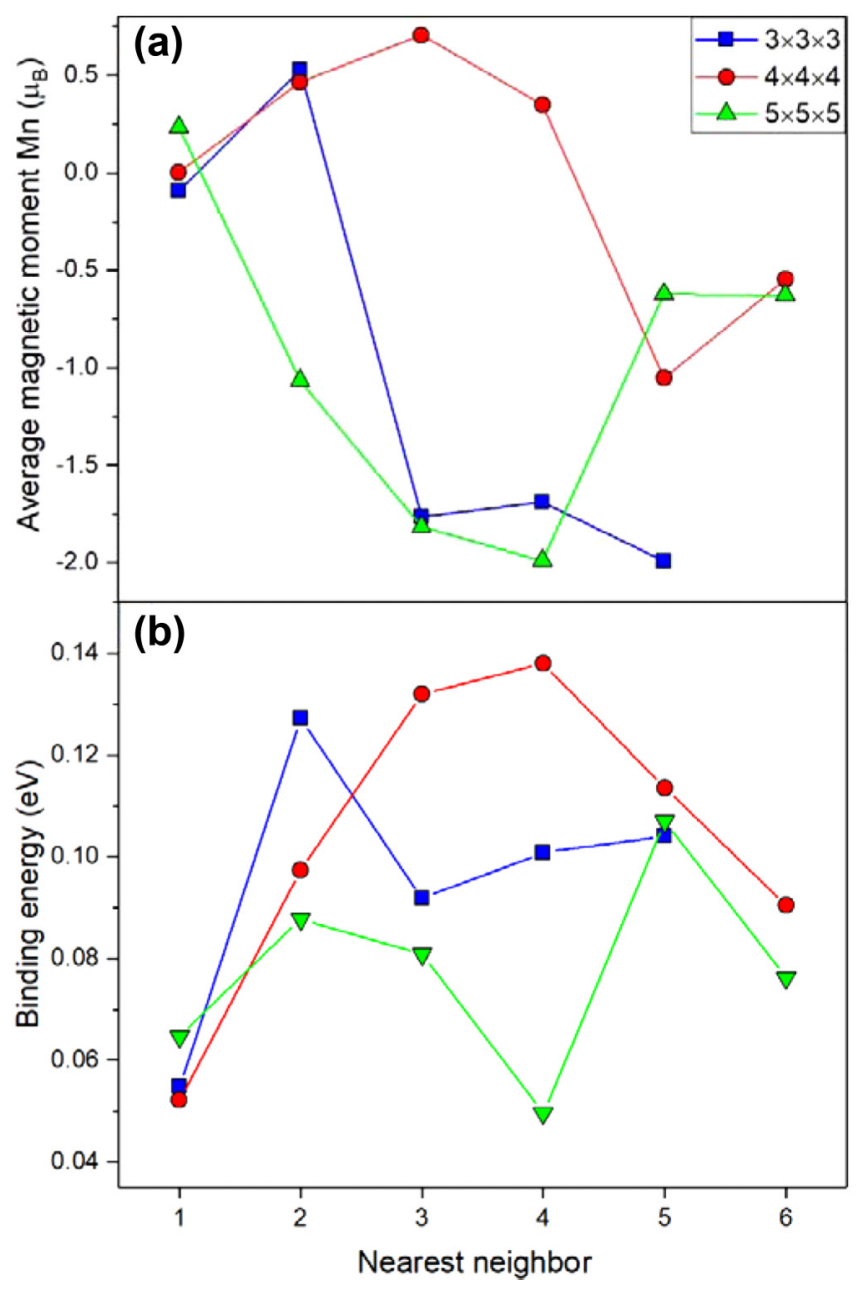

FIG. 13. (a) Average magnetic moment and (b) binding energy for $\mathrm{Mn}-\mathrm{Mn}$ nearest neighbors in $\alpha$-Fe.

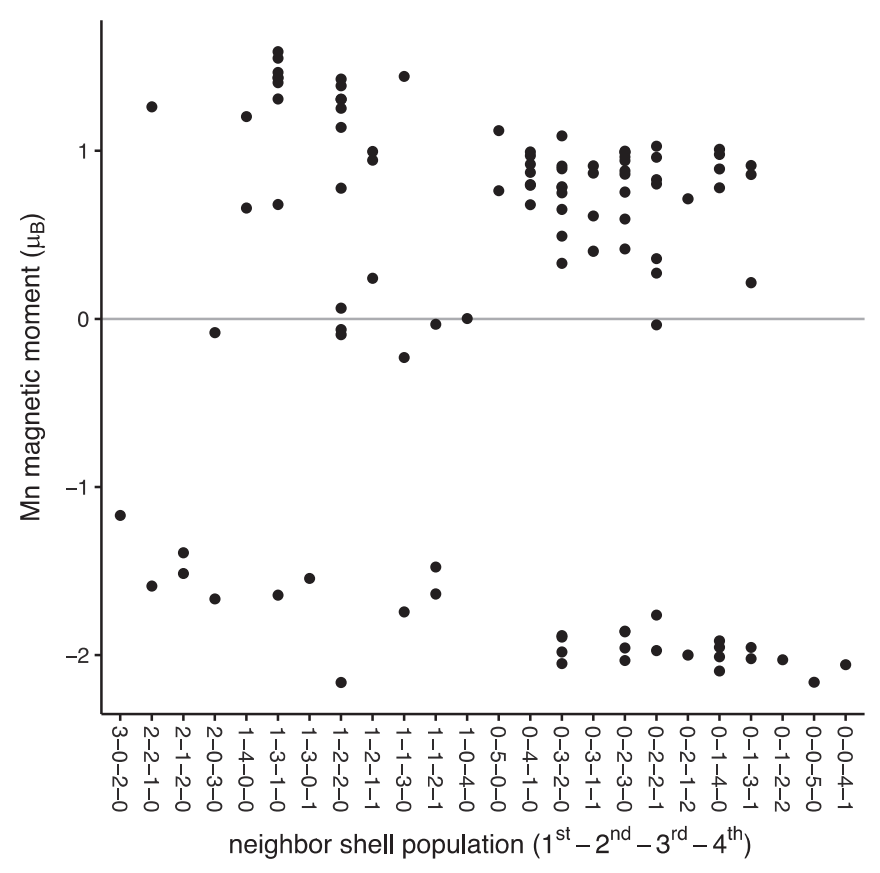

FIG. 14. Magnetic moment of each Mn atom for different configuration of the remaining five $\mathrm{Mn}$ atoms in supercell (located in shells 1-4). Configurations ordered from closest cumulative distance ("3-0-2-0" being three in the first shell, zero in the second, two in the third, and zero in the fourth), to furthest ("0-0-4-1": no Mn atoms in the first two shells, four in the third, and one in the fourth).

where $E_{\mathrm{Fe}}^{\text {bulk }}$ is obtained from a supercell of consistent size and dimensions as $E_{\mathrm{Fe}, \mathrm{Mn}}$.

The binding energies between two defects were calculated as follows:

$$
E_{\text {bind }}=\left(E_{D 1, D 2}+n_{\mathrm{Fe}} E_{\mathrm{Fe}}^{\text {bulk }}\right)-\left(E_{D 1}+E_{D 2}\right),
$$

where $E_{D 1, D 2}$ is the internal energy of the supercell containing both defects $D 1$ and $D 2$, and $E_{D 1}$ and $E_{D 2}$ is the internal energy of supercells (of consistent size and dimension with $E_{D 1, D 2}$ ) containing the respective defects.

\section{c. Vacancy and interstitial formation enthalpy}

In much the same method as calculating the substitution energy, the vacancy formation enthalpy is calculated as

$$
E_{\mathrm{vac}}=\left(E_{D}+E_{\mathrm{Fe}}^{\mathrm{bulk}}\right)-E_{P},
$$

where $E_{D}$ and $E_{P}$ are the defected and pristine supercells, respectively.

The interstitial formation enthalpy calculation was done as follows:

$$
E_{\mathrm{vac}}=E_{D}-\left(E_{P}+E_{\mathrm{Fe}}^{\mathrm{bulk}}\right)
$$

\section{Results}

When two $\mathrm{Mn}$ atoms were placed in 1 st to 6th nearest neighbor (nn) from each other in a $3 \times 3 \times 3,4 \times 4 \times 4$, and $5 \times 5 \times 5$ dimension supercell a stochastic behavior was found in the resultant magnetic moments of each Mn. Each adopted FM or AFM moments with varying magnitudes with no clear trend. 
The average magnetic moment and binding energies between the two Mn atoms are plotted in Fig. 13.

No correlation was found between spin on the Mn atom and the location of the remaining five $\mathrm{Mn}$ atoms in the cell, as illustrated in Fig. 14. Mn substitutions were found to retain both FM and AFM ordering (with respect to Fe), irrespective of the distance from other Mn atoms. However, a general trend is observed whereby the spin on $\mathrm{Mn}$ atoms decreases with increasing distance from neighboring $\mathrm{Mn}$ atoms.
[1] R. Ngayam-Happy, C. S. Becquart, C. Domain, and L. Malerba, J. Nucl. Mater. 426, 198 (2012).

[2] Y. Nagai, K. Takadate, Z. Tang, H. Ohkubo, H. Sunaga, H. Takizawa, and M. Hasegawa, Phys. Rev. B 67, 224202 (2003).

[3] H. J. Beattie and F. L. VerSnyder, Nature 178, 208 (1956).

[4] J. M. Vitek, Metall. Trans. A 18, 154 (1987).

[5] Y. Matsukawa, T. Takeuchi, Y. Kakubo, T. Suzudo, H. Watanabe, H. Abe, T. Toyama, and Y. Nagai, Acta Mater. 116, 104 (2016).

[6] G. R. Odette and G. E. Lucas, JOM 53, 18 (2001).

[7] R. Chaouadi and R. Gérard, J. Nucl. Mater. 345, 65 (2005).

[8] P. B. Wells, T. Yamamoto, B. Miller, T. Milot, J. Cole, Y. Wu, and G. R. Odette, Acta Mater. 80, 205 (2014).

[9] L. Malerba, C. S. Becquart, M. Hou, and C. Domain, Philos. Mag. 85, 417 (2005).

[10] C. S. Becquart, Nucl. Instrum. Meth. Phys. Res., Sect. B 228, 111 (2005).

[11] N. Soneda, in Materials Issues Generation IV Systems, edited by V. Ghetta, D. Gorse, D. Mazière, and V. Pontikis (Springer, Berlin, 2008), pp. 245-262.

[12] D. J. Hepburn, G. J. Ackland, and P. Olsson, Philos. Mag. 89, 3393 (2009).

[13] G. Bonny, D. Terentyev, A. Bakaev, E. E. Zhurkin, M. Hou, D. Van Neck, and L. Malerba, J. Nucl. Mater. 442, 282 (2013).

[14] D. Hobbs and J. Hafner, J. Phys.: Condens. Matter 13, L681 (2001).

[15] T. Yamada, N. Kunitomi, Y. Nakai, D. E. Cox, and G. Shirane, J. Phys. Soc. Jpn. 28, 615 (1970).

[16] J. Hafner and D. Hobbs, Phys. Rev. B 68, 014408 (2003).

[17] F. Boakye, K. G. Adanu, and A. D. C. Grassie, Mater. Lett. 18, 320 (1994).

[18] V. L. Moruzzi, P. M. Marcus, K. Schwarz, and P. Mohn, Phys. Rev. B 34, 1784 (1986).

[19] H. C. Herper, E. Hoffmann, and P. Entel, Phys. Rev. B 60, 3839 (1999).

[20] M. I. Mendelev, S. Han, D. J. Srolovitz, G. J. Ackland, D. Y. Sun, and M. Asta, Philos. Mag. 83, 3977 (2003).

[21] D. Bagayoko and J. Callaway, Phys. Rev. B 28, 5419 (1983).

[22] M. Pajda, J. Kudrnovsky, I. Turek, V. Drchal, and P. Bruno, Phys. Rev. B 64, 174402 (2001).

[23] V. T. Witusiewicz, F. Sommer, and E. J. Mittemeijer, J. Phase Equilib. Diffus. 25, 346 (2004).

[24] Y. Nakai and N. Kunitomi, J. Phys. Soc. Jpn. 39, 1257 (1975).

[25] H. Yamauchi, H. Watanabe, Y. Suzuki, and H. Saito, J. Phys. Soc. Jpn. 36, 971 (1974).

[26] P. Radhakrishna and F. Livet, Solid State Commun. 25, 597 (1978).

[27] P. Olsson, T. P. C. Klaver, and C. Domain, Phys. Rev. B 81, 054102 (2010).

[28] G. Kresse and J. Furthmüller, Phys. Rev. B 54, 11169 (1996).

[29] J. P. Perdew, K. Burke, and M. Ernzerhof, Phys. Rev. Lett. 77, 3865 (1996).
[30] G. Kresse and D. Joubert, Phys. Rev. B 59, 1758 (1999).

[31] M. Methfessel and A. T. Paxton, Phys. Rev. B 40, 3616 (1989).

[32] P. E. Blöchl, O. Jepsen, and O. K. Andersen, Phys. Rev. B 49, 16223 (1994).

[33] C. Varvenne, F. Bruneval, M. Marinica, and E. Clouet, Phys. Rev. B 88, 134102 (2013).

[34] E. Clouet, S. Garruchet, H. Nguyen, M. Perez, and C. S. Becquart, Acta Mater. 56, 3450 (2008).

[35] R. Nazarov, J. S. Majevadia, M. Patel, M. R. Wenman, D. S. Balint, J. Neugebauer, and A. P. Sutton, Phys. Rev. B 94, 241112 (2016).

[36] P. H. T. Philipsen and E. J. Baerends, Phys. Rev. B 54, 5326 (1996).

[37] J. Häglund, A. F. Guillermet, G. Grimvall, and M. Körling, Phys. Rev. B 48, 11685 (1993).

[38] S. Fujii, S. Ishida, and S. Asano, J. Phys. Soc. Jpn. 60, 1193 (1991).

[39] C. Kittel, Introduction to Solid State Physics (Wiley, New York, 2005).

[40] J. A. Oberteuffer and J. A. Ibers, Acta Cryst. B 26, 1499 (1970).

[41] C. B. Shoemaker, D. P. Shoemaker, T. E. Hopkins, and S. Yindepit, Acta Cryst. B 34, 3573 (1978).

[42] E. Rapoport and G. C. Kennedy, J. Phys. Chem. Solids 27, 93 (1966).

[43] E. Van Lenthe, J. G. Snijders, and E. J. Baerends, J. Chem. Phys. 105, 6505 (1996).

[44] L. Xiao and L. Wang, Chem. Phys. Lett. 392, 452 (2004).

[45] D. Hobbs, G. Kresse, and J. Hafner, Phys. Rev. B 62, 11556 (2000).

[46] K. Lejaeghere, V. Van Speybroeck, G. Van Oost, and S. Cottenier, Crit. Rev. Solid State Mater. Sci. 39, 1 (2014).

[47] E. Vincent, C. S. Becquart, and C. Domain, Nucl. Instrum Meth. Phys. Res., Sect. B 228, 137 (2005).

[48] D. Vanderbilt, Phys. Rev. B 41, 7892 (1990).

[49] B. Drittler, N. Stefanou, S. Blügel, R. Zeller, and P. H. Dederichs, Phys. Rev. B 40, 8203 (1989).

[50] V. I. Anisimov, V. P. Antropov, A. I. Liechtenstein, V. A. Gubanov, and A. V Postnikov, Phys. Rev. B 37, 5598 (1988).

[51] F. Kajzar and G. Parette, J. Magn. Magn. Mater. 14, 253 (1979).

[52] H. R. Child and J. W. Cable, Phys. Rev. B 13, 227 (1976).

[53] M. F. Collins and J. B. Forsyth, Philos. Mag. 8, 401 (1963).

[54] C. Freysoldt, B. Grabowski, T. Hickel, J. Neugebauer, G. Kresse, A. Janotti, and C. G. Van de Walle, Rev. Mod. Phys. 86, 253 (2014).

[55] P. A. Burr and M. W. D. Cooper, Phys. Rev. B 96, 094107 (2017).

[56] A. Bakaev, D. Terentyev, X. He, and D. Van Neck, J. Nucl. Mater. 455, 5 (2014).

[57] A. A. Mirzoev, M. M. Yalalov, and D. A. Mirzaev, Phys. Met. Metallogr. 101, 341 (2006). 
[58] N. I. Medvedeva, D. C. Van Aken, and J. E. Medvedeva, J. Phys.: Condens. Matter 23, 326003 (2011).

[59] D. L. Price and K. Sköld, in Neutron Scattering, edited by K. Sköld and D. L. Price (Academic Press, New York, 1986), pp. 1-97.

[60] J. W. Cable and Y. Tsunoda, Phys. Rev. B 50, 9200 (1994).

[61] Z. S. Basinski, W. Hume-Rothery, and A. L. Sutton, Proc. R. Soc. London, Ser. A 229, 459 (1955).

[62] J. Hubbard, Phys. Rev. B 20, 4584 (1979).

[63] P. Haas, F. Tran, and P. Blaha, Phys. Rev. B 79, 209902 (2009).

[64] R. W. Siegel, J. Nucl. Mater. 69, 117 (1978).

[65] M. Doyama, J. Nucl. Mater. 69-70, 350 (1978).

[66] M. L. Fullarton, R. E. Voskoboinikov, and S. C. Middleburgh, J. Alloys Compd. 587, 794 (2014).

[67] T. Garnier, M. Nastar, P. Bellon, and D. R. Trinkle, Phys. Rev. B 88, 134201 (2013).
[68] L. Messina, Z. Chang, and P. Olsson, Nucl. Instrum. Meth. Phys. Res., Sect. B 303, 28 (2013).

[69] L. Messina, L. Malerba, and P. Olsson, Nucl. Instrum. Meth. Phys. Res., Sect. B 352, 61 (2015).

[70] T. Ohnuma, N. Soneda, and M. Iwasawa, Acta Mater. 57, 5947 (2009).

[71] L. Messina, M. Nastar, T. Garnier, C. Domain, and P. Olsson, Phys. Rev. B 90, 104203 (2014).

[72] L. Messina, M. Nastar, N. Sandberg, and P. Olsson, Phys. Rev. B 93, 184302 (2016).

[73] H.-E. Schaefer, K. Maier, M. Weller, D. Herlach, A. Seeger, and J. Diehl, Scr. Metall. 11, 803 (1977).

[74] C.-C. Fu, F. Willaime, and P. Ordejón, Phys. Rev. Lett. 92, 175503 (2004).

[75] R. Armiento and A. E. Mattsson, Phys. Rev. B 72, 085108 (2005). 